\title{
Airport Simulation Based on Queuing Model Using ARENA
}

\author{
Da-Un Kim ${ }^{1}$, Min-Seok Jie ${ }^{2}$ and Won-Hyuck Choi ${ }^{3 *}$ \\ ${ }^{1}$ Department of Aeronautical Systems Engineering \\ ${ }^{2,3 *}$ Department of Avionics Engineering Hanseo University \\ ${ }^{1}$ kdu0011@naver.com, ${ }^{2} j i e m s @ h a n s e o . a c . k r,{ }^{3 *}$ choiwh@hanseo.ac.kr
}

\begin{abstract}
The check-in process for passengers using the airport takes a lot of time for departure and a considerable delay is delayed until the total boarding time. At the airport, a variety of systems are available to provide passengers convenience, such as self-check-in kiosks and automatic immigration screening, to assist passengers with rapid procedures. Therefore, this paper developed an airport simulation for efficient operation of unmanned systems such as self-check-in kiosks and automatic immigration screening. This simulation is a simulation until passengers enter the airport and pass through the security screening Point. The development tool was developed using ARENA Simulation and modeled using queuing theory. Based on the developed simulation results, the airport can establish an unmanned system operation plan of the airport.
\end{abstract}

Keywords: Queuing, Unmanned System, ARENA, Airport Operation, Airport Simulation

\section{Introduction}

Due to the increase in low-cost airlines, the number of travelers to the airport is steadily increasing. In the case of domestic airports, the number of tourists visiting the country is increasing due to the popularity of Korean Wave and shopping tourism. In addition, new routes are increasing in China and Southeast Asian countries, especially low-cost airlines, and the growth of airport use is expected to continue. Due to the increase in the number of tourists, it is expected that the problem of the adequate capacity of airport facilities and various difficulties in operation of airport facilities are expected [1].

At the airport, an waiting situation is essential for boarding the aircraft. Passengers must wait until they arrive at the airport and boarding the aircraft. When passengers continue to be in the waiting situation, the airports become increasingly crowded and passengers become increasingly uncomfortable. To solve this situation, the airports are preparing various measures. For example, expansions of security search facilities, introduction of smart airports, expansion of parking lots, expansion of convenience facilities, and expansion of unmanned systems. In this paper, the simulation of the unmanned system is carried out [2][3].

The operation of the unmanned system applied in the simulation model development was applied to self-check - in and self - baggage consignment. Passengers enter the airport and walk to the unmanned system, use the unmanned system, travel to the security screening point, and pass through the security screening point. Also, when the number of unmanned systems is small, it causes a long queue and waits time, and it needs a large space to accommodate it. The number of unmanned systems depends on the number of departing passengers and the average processing time per passenger. Also, the

Received (December 13, 2017), Review Result (March 14, 2018), Accepted (March 20, 2018)

* Corresponding Author 
convenience and psychological factors of passengers should be considered. The number of unmanned systems should be able to accommodate the maximum queue. The service time of the operation of the unmanned system affects the number of unmanned systems and the space required. The longer the service time, the more space is required to accommodate more waiting passengers, or more unmanned systems are required to reduce standby time [4][5].

This paper is based on the queuing theory and developed the airport simulation using ARENA Simulation. Simulations for applying the unmanned system to the airport were developed, adapted to the airport characteristics, and the optimal unmanned system was analyzed. Simulation was designed directly for accurate analysis. The design method was designed using ARENA Simulation and modeled using queuing theory [6].

\section{Queuing Theory}

Queues are seen as part of a much generalized life wherever services are provided. A queue is a queue of consumers waiting to receive service from server. In any service system, a queue occurs when the current demand exceeds service capability. When a service provider is too busy or the number of servers is low, a customer who arrives immediately receives a service and a queue occurs. The economic value of waiting is cost for the supplier, and it is money for consumers [7].

The occurrence of queues in the service system has a great impact on the business side. The cost of providing the waiting space, or the loss of commerce that may occur if the customer refuses to leave the queue or waits before receiving the service, or loss of credibility and customer satisfaction, Congestion can result from business operations or disturbances to customers. The ultimate goal of queue management is to minimize the total cost, which is the sum of the cost of the customer waiting for the service and the cost associated with the service capacity [8].

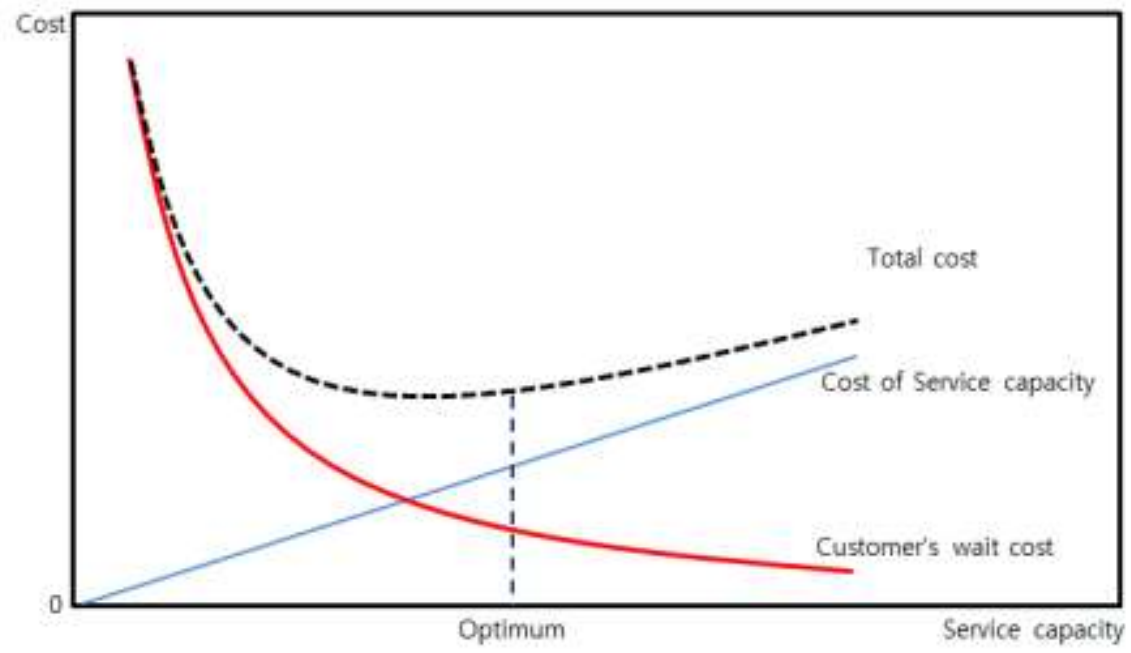

Figure 1. Total Cost $=$ Customer’s Wait Cost + Service Capacity Cost

\subsection{Characteristics of Queues}

There are several characteristics of queues. The population source is divided into infinite source and finite source. The infinite source situation is a characteristic that the arrival of customers is not limited, and the finite source situation is a characteristic in which the number of potential customers is limited. The number of service windows is divided into a single window and a plurality of windows. A single window can only be processed by one customer at a time on one channel, there are more than one service 
channel in multiple windows. In general, the arrival and service patterns assume that arrival and service rates follow Poisson distribution or exponential distribution. The relationship between rate and time is a inverse relationship. That is, the arrival rate is the inverse of the arrival interval time and the service rate is the inverse of the service time. When service time follows exponential distribution, service rate follows Poisson distribution. Similarly, if the customer's arrival rate follows the Poisson distribution, the customer's arrival interval follows the exponential distribution. The order in which customers are generally applied is on a first in, first out. Finally, the measure of queue performance is the average number of customers waiting in the system, the average wait time that a customer waits in the system, system utilization, which is the rate at which production capacity is used, implicit costs due to a given capacity and its associated queue, And the probability that the arriving customer should wait to receive the service.

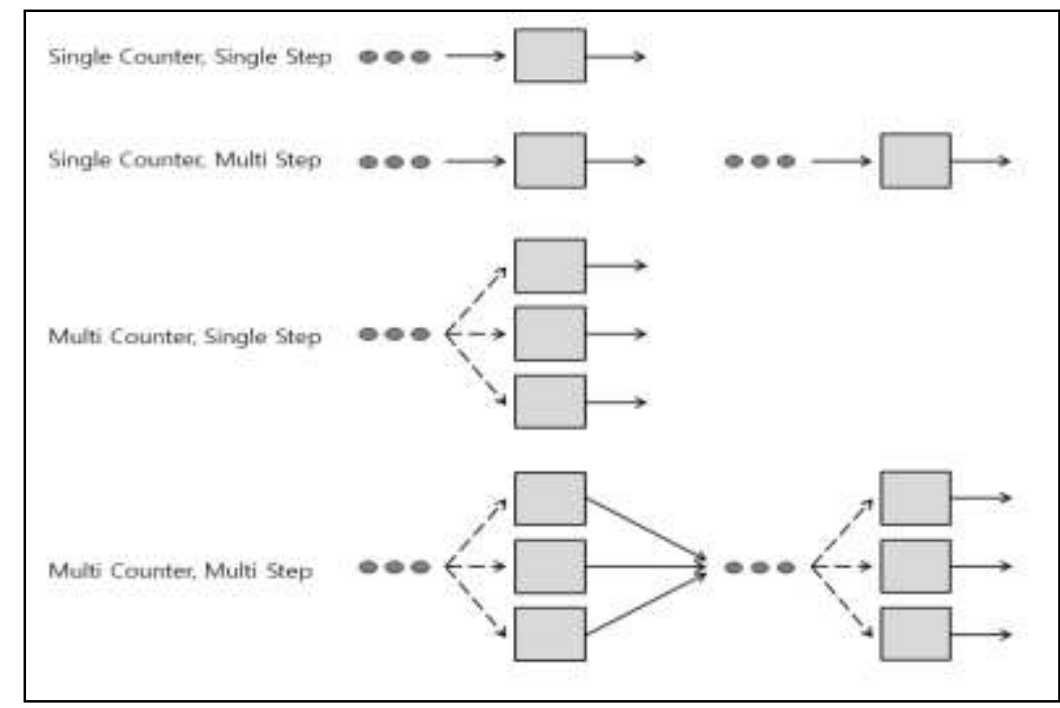

Figure 1. Four Common Forms of Queuing Systems

\subsection{Modeling of Queuing Theory}

$$
\begin{aligned}
& P_{n}=\left(1-\frac{\lambda}{\mu}\right)\left(\frac{\lambda}{\mu}\right)^{n} \\
& L_{s}=0 * P_{0}+1 * P_{1}+2 * P_{2}+\cdots+n * P_{n}=\frac{\lambda}{\mu-\lambda} \\
& L_{q}=\frac{\lambda}{\mu-\lambda}-\frac{\lambda}{\mu}=\frac{\lambda^{2}}{\mu(\mu-\lambda)} \\
& W_{q}=L_{q} * \frac{1}{\lambda}=\frac{\lambda}{\mu(\mu-\lambda)} \\
& W_{s}=W_{q} \frac{1}{\mu}=\frac{1}{\mu-\lambda} \\
& \rho=\frac{\lambda}{\mu}
\end{aligned}
$$

Where $\mu$ the average is service rate and $\lambda$ is the average arrival rate. The $P_{n}$ in equation (1) represents the probability that there are $\mathrm{n}$ customers in the system. $L_{s}$ in equation (2) represents the average number of customers in the system. $L_{q}$ in equation (3) represents the length of the queue. $W_{q}$ in equation (4) represents the average waiting time. $W_{s}$ in equation (5) represents the average delay time in the system. $\rho$ in Equation (6) represents the service utilization rate [9]. 


\section{Simulation Configuration}

ARENA has both ease of use and flexibility of simulation language that can be found in advanced simulators. If user wants, user can even have the flexibility of a general purpose procedural language such as the Visual Basic programming system or C. ARENA provides graphical simulation modeling and modules templates that can be combined to build a wide variety of simulation models. For ease of display and configuration on the computer screen, modules are grouped into panels to form templates. By changing the panel, the user can call up a completely different combination of simulation modeling components and functions. In most cases, user can gather modules from multiple panels and use them in one model [10][11].
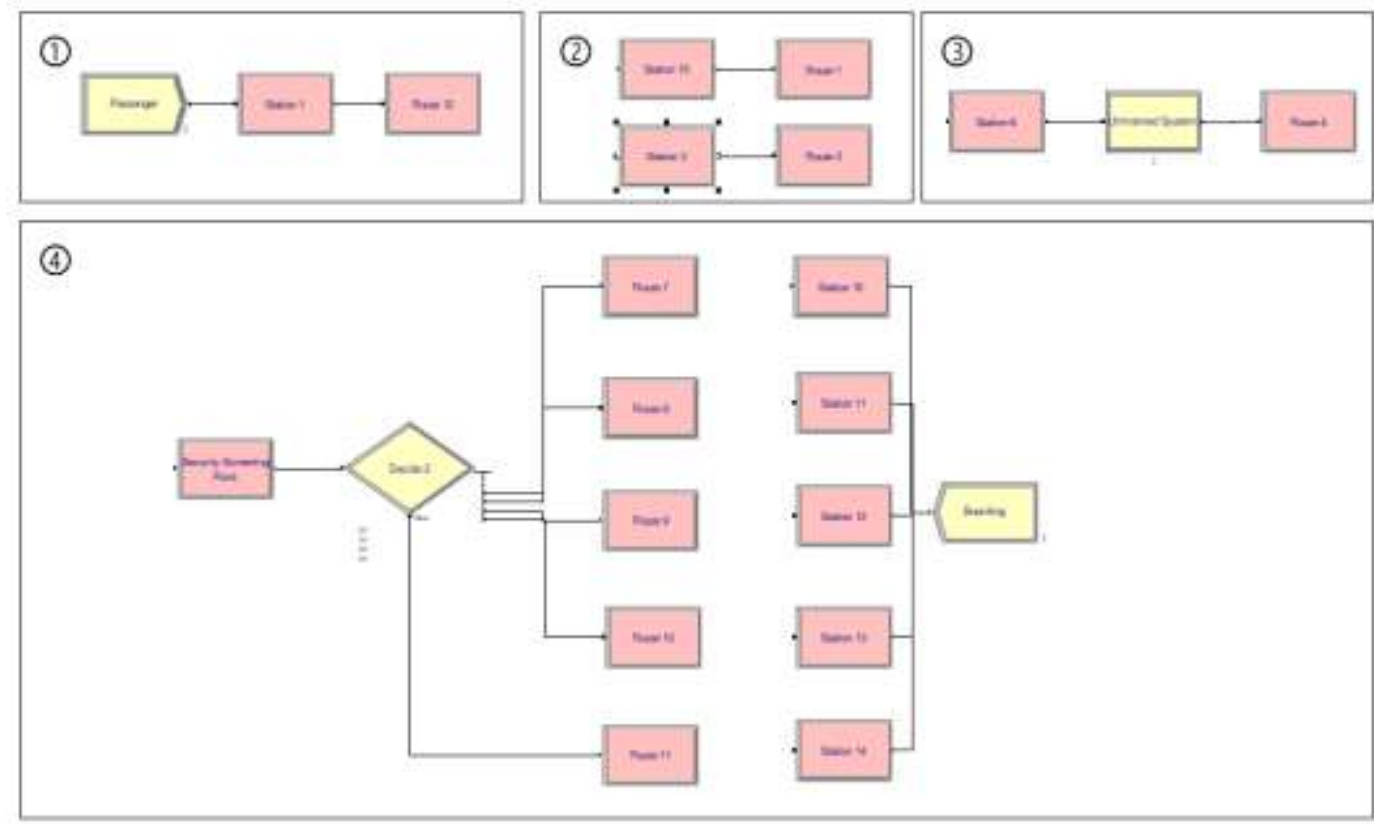

Figure 3. Simulation Diagram

Figure 3. is a simulation diagram. (1) is a part that creates a passenger and enters into the simulation. (2) is the part where passengers who entered are moving to the unmanned system. (3) is the part where passengers use the unmanned system. (4) is the part where passengers who use the unmanned system pass through the security screening point.

\subsection{Arena Simulation Module}

\begin{tabular}{|c|c|c|c|c|}
\hline Create & & & $?$ & $\times$ \\
\hline Name: & & \multicolumn{3}{|c|}{ Entity Type: } \\
\hline Passenger & & $\checkmark$ & by passenger & $\checkmark$ \\
\hline $\begin{array}{l}\text { Time Between Arrivals } \\
\text { Type: }\end{array}$ & Value: & & Units: & \\
\hline Random (Expo) $\checkmark$ & 10 & \multicolumn{2}{|r|}{ Minutes } & $v$ \\
\hline Entities per Arrival: & Max Arrivals: & \multicolumn{2}{|r|}{ First Creation: } & \\
\hline 10 & 1000 & \multicolumn{2}{|r|}{10} & \\
\hline & OK & \multicolumn{2}{|c|}{ Cancel } & \\
\hline
\end{tabular}

Figure 4. Passenger Creation Module 
In the Type of Time between Arrivals area, Random (Expo) is selected, which means that the arrival time interval will be generated according to the extraction of the random variable value, specifically the exponential distribution. Enter 10 in the Value field and Minutes in the Units field, which means 10 minutes. In the last line of the dialog box, we have entered 10 for the Entities per Arrival value, which means that 10 people are created each time it is created. Max Arrivals specifies 1000, and First Creation arrives 10 minutes after arriving first.

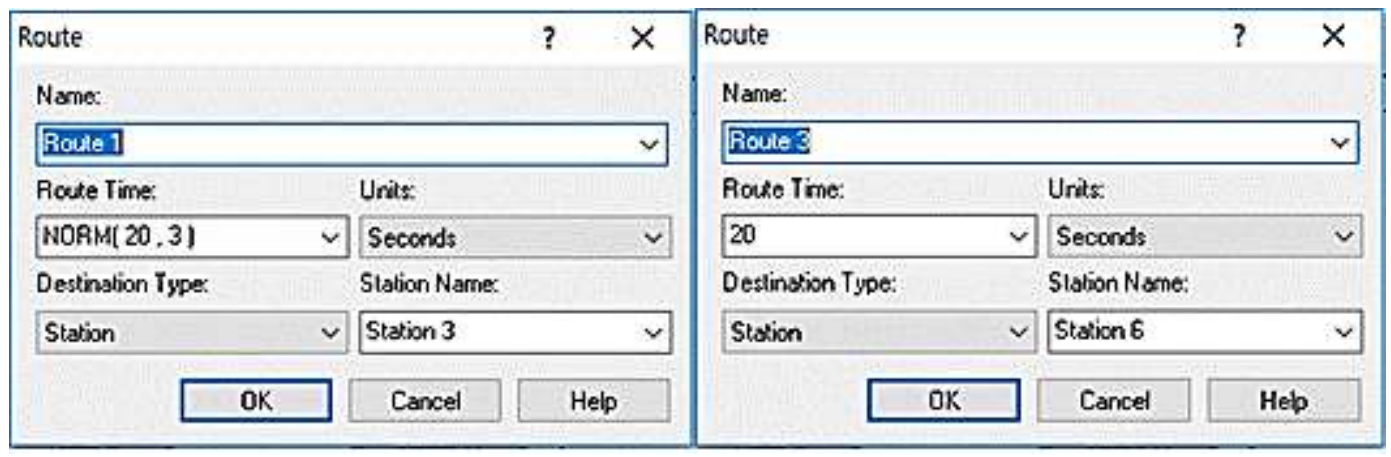

Figure 5. Passenger Moving Module

Passengers created in the Creation Module move to the unmanned system. At this time, to give a difference in moving speed for each person, an average of 20 and a standard deviation of 2 were set for each passenger, and the unit was set to seconds. Passengers arriving in front of the unmanned system enter the line. In this case, it is set to 20 seconds because all of them must move at the same speed. In other words, passengers enter the airport and go to the unmanned system through 20 seconds $\pm \alpha+20$ seconds.

Table 1. Process Module Setting

\begin{tabular}{|ll|}
\hline $\begin{array}{l}\text { Name } \\
\text { Action }\end{array}$ & $\begin{array}{l}\text { Unmanned System } \\
\text { Seize Delay Release }\end{array}$ \\
\hline \multicolumn{2}{|c|}{ Resources } \\
Type & Resource \\
Resource Name & Resource 1 \\
Quantity & 1 \\
\hline Delay Type & Normal \\
Units & Minutes \\
Value(Mean) & $2+3$ \\
Std Dev & 1 \\
\hline
\end{tabular}

Table 1 shows the setting values of the Process module of the unmanned system, and is used to set the time required for resources, queues, and entities. We chose Seize Delay Release for Action, which means that in this module, entities will occupy a few units of resources, delaying the service time and letting that resource be released, allowing other entities to occupy this resource. You can specify a value in the Priority field to prioritize the entities that want to acquire resources. In Arena, a smaller value represents a higher priority. Normal (Normal Distribution) is used in the list of Delay Type. Minutes are defined in the Units field and Value Added is selected in the Allocation field. Value (Mean) value is $2+3$, which means the time of self-check-in and self-baggage consignment. That is, those who use the unmanned system will exit after a normal distribution time of 5 minutes and a standard deviation of 1 minute. 


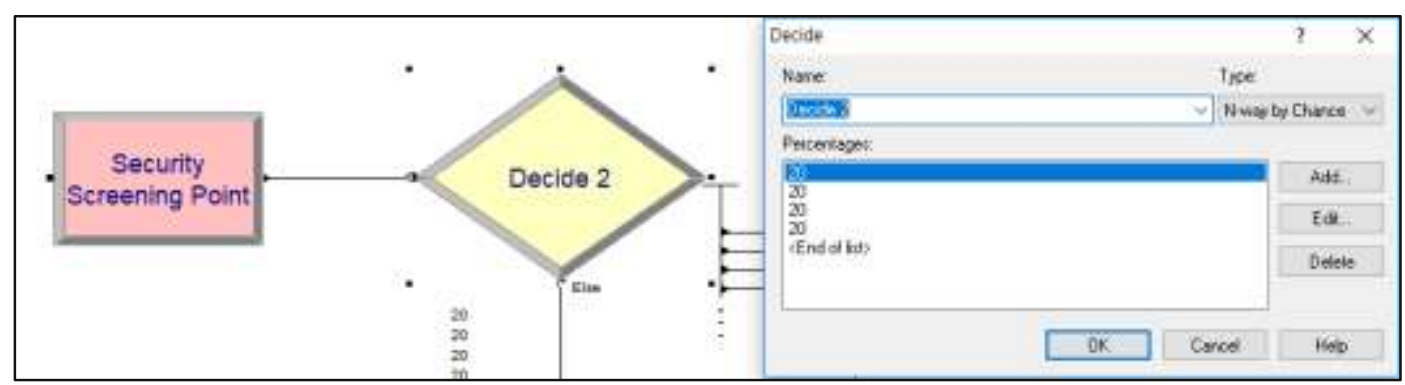

Figure 6. Passenger Moving Module

Finally, passengers who use the unmanned system go to the security screening point. At this time, an average of 20 and a standard deviation of 2 were set for each passenger, and the unit was set to seconds. There are 5 security screening points, and the probability of passengers entering each security screening point is $20 \%$. Passengers traveling to the front of the search area will pass the security screening point for one minute. Passengers traveling to the security screening point pass a security screening point for one minute. Passengers who have passed through the security screening point are logged in Entity Statistics.

\subsection{Run Setup}

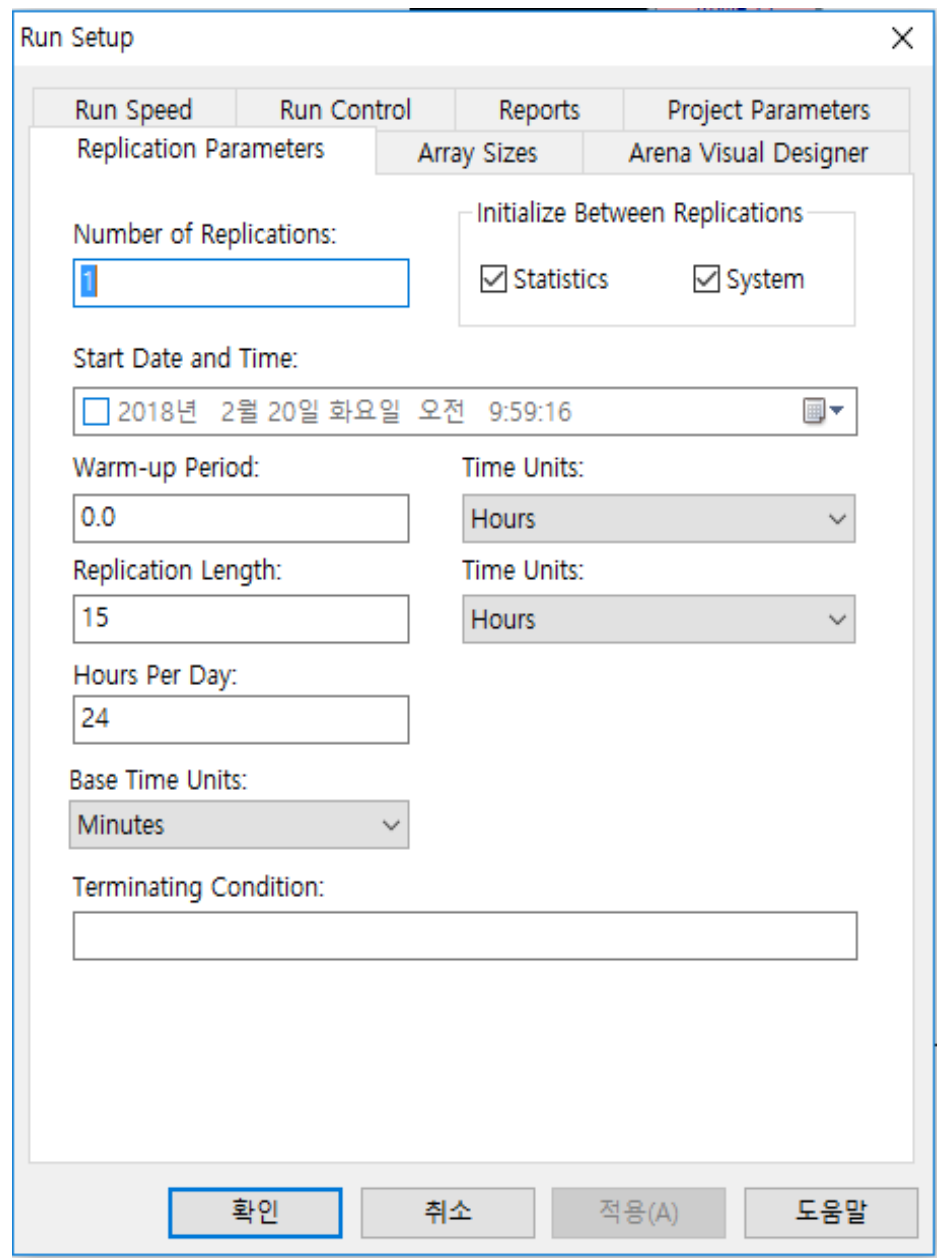

Figure 7. Run Setup of Replication Parameters 
In the Replication Parameters tab of Run Setup, you can control various aspects of execution. Number of Replications used the default value of 1. The Start Date and Time fields are used as default values. This field is used to set simulation time 0 to a specific date and time on the calendar. The warm-up period is the time at which statistical values collected from the start of the simulation execution are erased to remove the influence of the irregular initial state on the statistics. Length of Replication is specified as 15 , and its time unit is specified as hours. The Hours Per Day field defaults to 24, the default value. The Base Time Units field is used not only to report time-based output, but also to specify the time unit Arena uses by default when interpreting any time-based numeric input value without a Time Unit field. The Terminating Condition field is used to set up a simulation termination rule that is complex or state dependent. This is used when all objects in the model are emptied or when you want to end the simulation after a certain amount of time has elapsed.

In all Arena models, the simulation end condition must be specified. This is an important part of modeling. Arena does not provide a default termination condition because it does not know what the user wants. If the user does not explicitly specify the end-of-simulation condition, the simulation will run forever or until the user intervenes to stop it.

In other words, the simulation is to conduct simulation for 15 hours based on 24 hours a day.

\section{Simulation}

Simulations were conducted to verify the efficient number of unmanned systems. If the number of unmanned systems is $1 \sim 5$, the simulation cannot process all the passengers who continue to enter the system, and the system continues to accumulate in the queue. As a result, the number of passenger's divergence has appeared. Therefore, the simulation has been carried out from the unmanned system 6, and the simulation time is 15 hours.

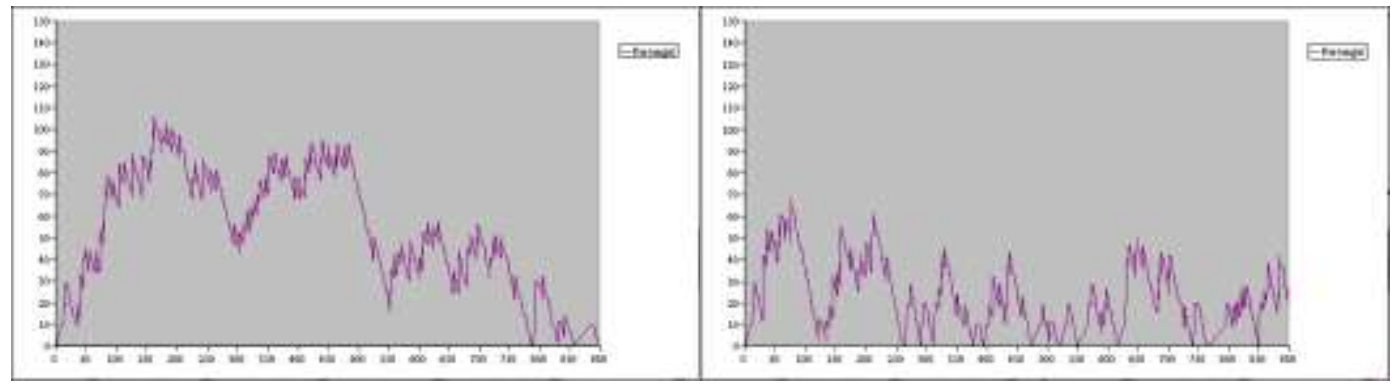

Figure 8. WIP(Work In Process) Entity of Unmanned System 6, 7

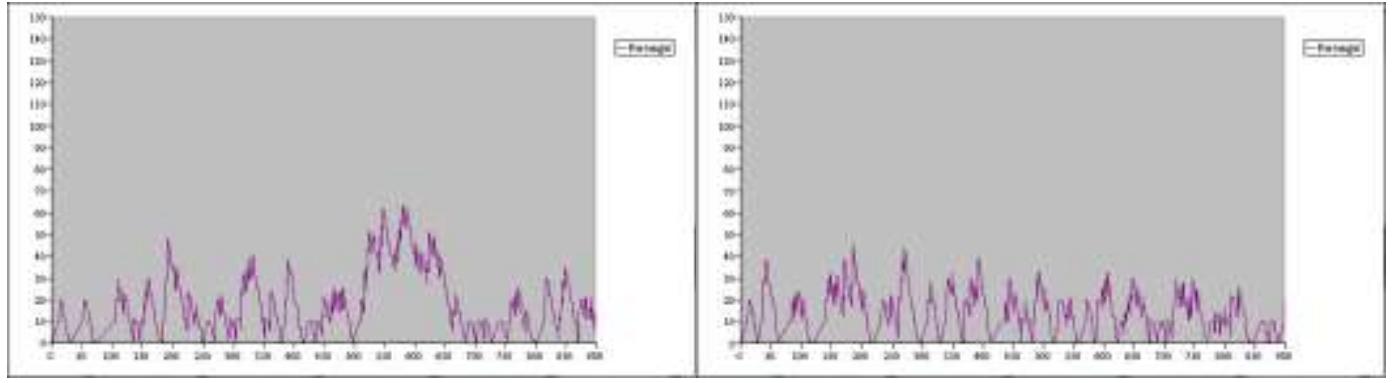

Figure 9. WIP Entity of Unmanned System 8, 9 


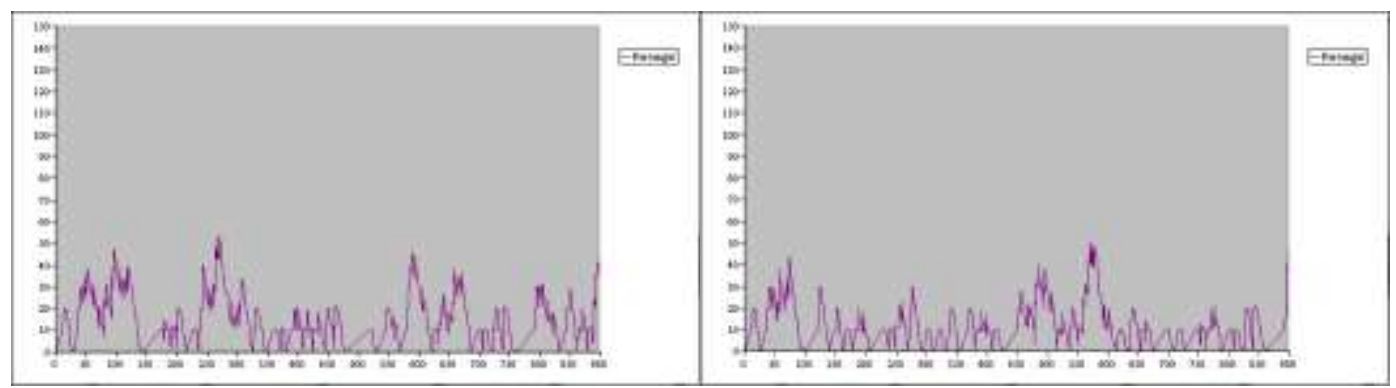

Figure 10. WIP Entity of Unmanned System 10, 11

Figure 8, 9, and 10 show the number of passengers in the system during the simulation. As the number of unmanned systems increases, the number of passengers in the system decreases. However, the graphs of Unmanned Systems 10 and 11 in Figure 10 show similar trends.

Table 2. Report of Entity

\begin{tabular}{|l|l|l|l|}
\hline $\begin{array}{l}\text { Unmanned } \\
\text { System }\end{array}$ & $\begin{array}{l}\text { Wait Time } \\
\text { Average }\end{array}$ & $\begin{array}{l}\text { Wait Time } \\
\text { Maximum }\end{array}$ & $\begin{array}{l}\text { Total Time } \\
\text { A verage }\end{array}$ \\
\hline $\mathbf{6}$ & 39.4446 & 77.8286 & 48.0548 \\
\hline $\mathbf{7}$ & 10.9557 & 40.5147 & 19.5551 \\
\hline $\mathbf{8}$ & 7.3647 & 30.9003 & 15.8422 \\
\hline $\mathbf{9}$ & 3.2662 & 17.0904 & 11.8042 \\
\hline $\mathbf{1 0}$ & 3.1021 & 13.8764 & 11.6547 \\
\hline $\mathbf{1 1}$ & 1.9315 & 12.9578 & 10.4818 \\
\hline
\end{tabular}

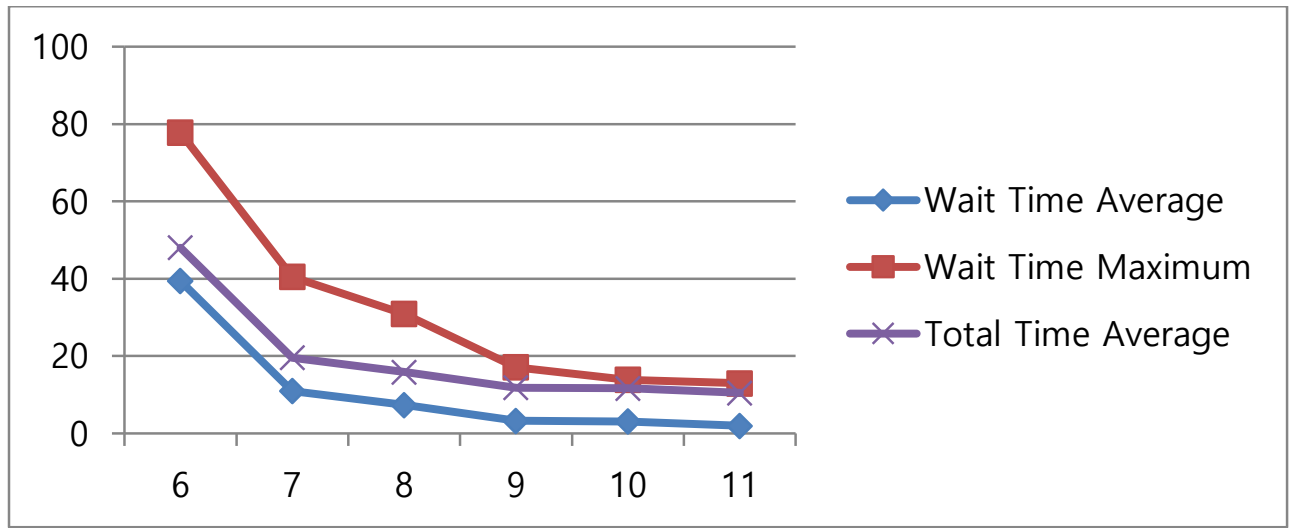

Figure 11. Entity Graph

Table 2 and Figure 11 show the time spent waiting for passengers to use the unmanned system and the total time to complete the procedure after entering the airport that when passenger data is set as in Chapter 3. When the number of unmanned systems is 6 , passengers should wait for 40 minutes on average. The longest waiting passengers waited for 77 minutes and were able to complete the check-in process. As the number of unmanned systems increases, the number of passengers waiting and the total time spent at the airport decreases. However, it can be seen that the amount of decrease is gradually decreasing.

When the number of unmanned systems is $9,10,11$, the total time average of passengers is almost the same. However, when the number of unmanned systems is 9, the Wait Time Maximum is slightly higher. 


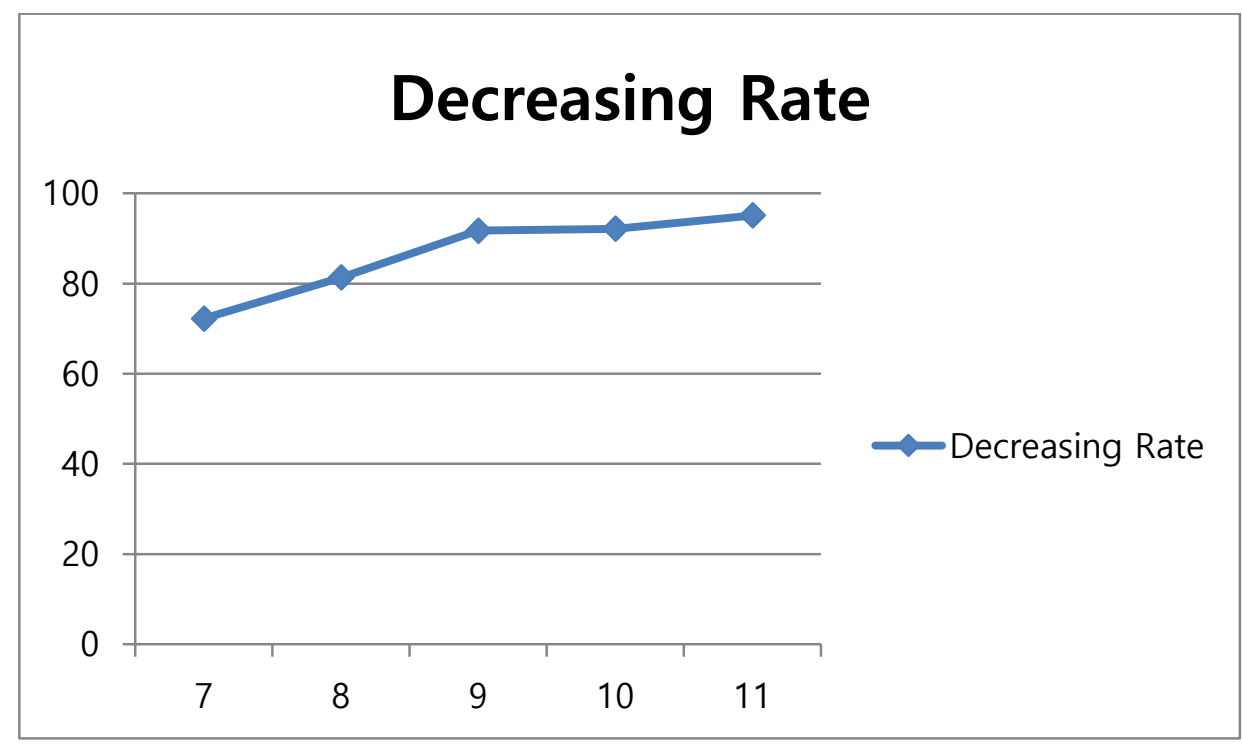

Figure 12. Degreasing Rate of Wait Time Average

Figure 12 shows the reduction rate of the Wait Time Average. When the number of unmanned systems is 9 , the reduction rate is over $90 \%$, which is the most efficient. But beyond that, we cannot expect more efficiency. Therefore, the total number of unmanned systems is 9 , which is the most efficient, but considering the Wait Time Maximum, we can consider 10 .

\section{Conclusion}

In order to reduce the waiting time and discomfort index of passengers using the airport, self-service is expanding at the airport. Unmanned systems are systems that satisfy these services. This paper developed an airport simulation for efficient operation of unmanned systems such as self-check - in kiosks and automatic immigration screening. We have modeled using the queuing theory and confirmed the result that the congestion is lowered by reducing the waiting time through the direct designed simulation. By reducing the waiting time, the discomfort of the passengers can be reduced, and as a result of the simulation, the airport and the airline can efficiently operate the unmanned system. However, the data values of the passengers applied to the current simulation are all arbitrary data. Passenger data collection should be preceded in order to obtain more reliable results.

\section{Acknowledgments}

This paper is a revised and expanded version of a paper entitled [Airport Simulation Development Using Arena] presented at [ICASC 2018, Amari Watergate, Bangkok, Thailand last January 18-21].

This paper was supported by 2017 research grant from Hanseo University.

\section{References}

[1] G. U. Ryu, Editor, “Airport Operation and Management”, BAEKSANS PUBLISHING, Korea, (2004).

[2] J. H. Cho and D. K. Lee, "Airport Punctuality Analysis Using Multi-Dimensional Visual Analysis Method", Journal of the Korea Society Conference 2010 IT services, (2010), pp. 285-290.

[3] G. R. Kim and W. J. Yhang, "The Effect of the Waiting Environment on the Perceptions of the Waiting Time and the Crowding", Korea Journal of Hotel Administration, vol. 22, no. 3, (1990), pp. 235-248.

[4] S. J. Lee, K. S. Lee and W. Y. Kim, "A Study on the Application Method of Automated Check-in Procedures in Korea through Overseas Case Analysis", Aviation Management Society of Korea, vol. 2014, no. 0, (2014), pp. 508-512. 
[5] C. H. Shin and H. J. Lee, "A Study on Airlines` Self-Service Technology (SST) Quality Affecting Customer Satisfaction and Behavioral Intention", Tourism Management Research, vol. 16, no. 2, (2012), pp. 97-117.

[6] J. C. Park, K. T. Kim and H. Y. Youn, "Implementation of Traffic Prediction System Based on Queuing Network Model", Proceedings of the Korean Society of Computer Information Conference, vol. 22, no. 2, (2014), pp. 395-396.

[7] H. W. Lee, Editor, "Queuing Theory - Stochastic Process Analysis", Sigmapress, Korea, (2006).

[8] K. H. Son, H. A. Kim, E. J. Kim, D. U. Han and S. T. Kim, "A simulation Study on a Bank Telling System by Using Arena", Korean Journal of Business Administration, no. 27, (2001), pp. 81-104.

[9] S. S. Lee and H. W. Lee, "Modeling of Vacation Queues by Supplementary Variable", Journal of the Korean Institute of Industrial Engineers, vol. 16, no. 1, (1990), pp. 107-114.

[10] M. D. Rossetti, "Simulation Modeling and Arena", WILEY, United States, (2016).

[11] W. David Kelton, R. P. Sadowski and N. B. Zupick, "Simulation with ARENA", MC Graw Hill Education, United States, (2015).

\section{Authors}

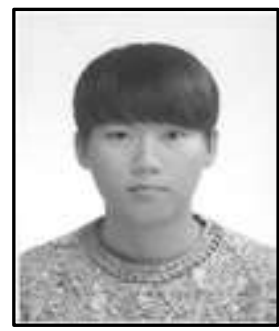

Da-Un Kim, graduate of the Hanseo University avionics bachelor's and a master's degree and Aviation Systems Engineering in 2015.

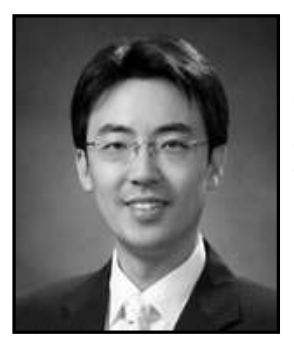

Min-Seok Jie, graduated from the Ph.d degree in the department of avionics Engineering from Korea Aerospace University in 2006. he is Hanseo University Avionics Engineering Professor in to the present in 2008.

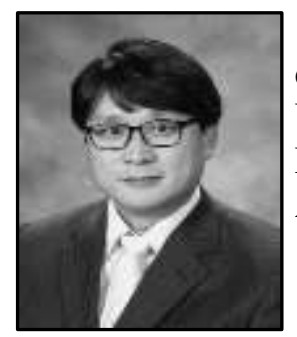

Won-Hyuck Choi, graduated from the Ph.d degree in the department of avionics Engineering from Korea Aerospace University in 2006. the Doowon Technical University Smart Network Professor from 2008 to 2014. he is Hanseo University Avionics Engineering Professor in to the present in 2014. 